\title{
Factores pronósticos de mortalidad en niños con bacteriemias adquiridas en la comunidad (BAC) hospitalizados en un centro de referencia del Paraguay
}

\author{
Prognostic mortality factors in children with acquired \\ bacteremia in the community $(A B C)$ hospitalized at a \\ tertiary medical center in Paraguay
}

\author{
Soraya Araya ${ }^{1,2}$, Avelina Troche', Sara Amarilla', Gabriela Sanabria', Claudia Zarate', Fernando \\ Galeano', Rocío Benitez', Sandra López', Juan Irala', Silvio Apodaca' y Antonio Arbo ${ }^{1,3}$
}

\section{RESUMEN}

Objetivo: Identificar etiologìa de BAC y factores pronósticos de mortalidad. Material y Método: Estudio retrospectivo de BAC en pacientes de 29 días a 16 años hospitalizados en un Centro de Referencia del Paraguay, periodo 2007 a 2015, se analizaron variables demográficas, clínicas y laboratoriales categorizando a los pacientes según el desenlace (Vivos o Muertos) Resultados: 187 casos de BAC , edad media $40 \pm 35$ meses, M/F 1,4:1, edad < 1 año: 30,5\% (57/187), 1 a 4 años 49,2\% (92/187), 5 a 10 años 15,5\% (29/187) y > 10 años 4,8 \% (9/187). Diagnósticos de ingreso: Neumonía (78\%), Infección de Piel y Partes Blandas (9\%), Infección a Múltiples focos $(7 \%)$, Meningitis (3,7\%) Bacteriemia sin foco $2 \%$. Las bacteriemias por Gram Positivos fueron más frecuentes que las bacteriemias por Gram Negativos: 91,4 vs 8,6\% ( $<<0,05)$, aislamientos: S.pneumoniae, S.aureus, S.coagulasa negativo ( $2 / 2)$ y K. pneumoniae $(46 \%, 34 \%, 7,5 \%$ y $2 \%)$. Ingresaron a UCI 63 pacientes $(33,7 \%)$, mortalidad 16,5\%. Variables asociadas a mortalidad: Edad $<5$ años $(\mathrm{p}<0,05)$, Desnutrición $(\mathrm{p}=0,02, \mathrm{RR}=2,4, \mathrm{IC} 95 \%: 1,1-5)$, Infección a múltiples focos( $\mathrm{p}=0,002, \mathrm{RR}=3,2$, IC 95\%: 1,6-6,4), Choque $\mathrm{p}<0,001$, RR19, IC95\%:7,8-46), Glasgow $<12$ ( $<<0,001$, RR=5,5, IC 95\% :3,2-9,6), Sat O2 < 94\% ( $\mathrm{p}=0,007$, RR 2, IC95\%: 1,1-4) , Hb< 7 ( $\mathrm{p}<0,01$, RR: 11, IC95\%: 6,8 a 19), GB > 15000, plaquetas $<100$ $000(p<0,001, R R=4,4$, IC95\%: 2,4- 7,8), Bacteriemia por Gram Positivos ( $p=0,001, R R=4,9$, IC 95\%: 1,5-15). Conclusión: En niños con BAC son más frecuentes las bacteriemias por Gram positivos. Se identificaron factores pronósticos de mortalidad

Palabras Clave: Bacteriemia, niños, mortalidad.

\section{ABSTRACT}

Objective: To identify the etiology of $\mathrm{ABC}$ and prognostic factors of mortality. Materials and Methods: This was a retrospective study of $A B C$ in patients aged 29 days to 16 years who were hospitalized at a Tertiary Medical Center in Paraguay, during the period from 2007 to 2015. We analyzed demographic, clinical and laboratory variables, categorizing the patients according to the outcome (Survival or Death). Results: We identified 187 cases of ABC, with an average age of $40 \pm 35$ months, $\mathrm{M} / \mathrm{F}$ ratio of $1.4: 1$, age $<1$ year: $30.5 \%$ (57/187), 1 to 4 years $49.2 \%$ (92/187), 5 to 10 years $15.5 \%$ $(29 / 187)$ and $>10$ years $4.8 \%$ (9/187). The admission diagnoses were: Pneumonia (78\%), Skin and Soft Tissue Infection (9\%), Multiple Source Infection (7\%), Meningitis (3.7\%) and Bacteremia without a source $2 \%$. Gram-positive bacteremia was more frequent than gram-negative bacteremia, 91.4 vs. $8.6 \%$ ( $p<0.05)$, isolates were: S.pneumoniae, S.aureus, S.coagulase negative $(2 / 2)$ and $\mathrm{K}$. pneumoniae $(46 \%, 34 \%$, $7.5 \%$ and $2 \%$, respectively). 63 patients $(33.7 \%)$ were admitted to the Intensive Care Unit (ICU), the mortality rate was $16.5 \%$. Variables associated with mortality were: Age $<5$ years ( $p$ $<0.05$ ), Malnutrition ( $p=0.02, \mathrm{RR}=2.4,95 \% \mathrm{CI}$ : 1.1-5), infection with multiple sources ( $\mathrm{p}=0.002, \mathrm{RR}=3.2,95 \% \mathrm{CI}$ : 1.6-6.4) ), Shock ( $\mathrm{p}<0.001, \mathrm{RR}=19,95 \%$ : 7.8-46), Glasgow coma scale score $<12$ ( $\mathrm{p}<0.001, \mathrm{RR}=5.5,95 \%$ 95\% CI: 3.2-9.6), O2 Sat $<94 \%$ ( $p=0.007$, RR 2, 95\% CI: 1.1-4), Hb $<7$ ( $<<0.01$, RR: 11, 95\% CI: 6.8 to 19), White Blood Cell count $>15000$, platelets $<100000$ (p $<0.001, \mathrm{RR}=4.4,95 \% \mathrm{CI}: 2.4-7.8)$, Gram-positive bacteremia ( $\mathrm{p}$ $=0.001, \mathrm{RR}=4.9,95 \%$ CI: 1.5-15). Conclusions: Gram-positive bacteremia is more frequent in children with ABC. Prognostic factors of mortality were identified.

Keywords: Bacteremia, children, mortality.

${ }^{1}$ Instituto de Medicina Tropical. Asunción Paraguay.

${ }^{2}$ Universidad Católica de Asunción, Asunción, Paraguay.

${ }^{3}$ Universidad Nacional de Asunción, San Lorenzo, Paraguay.

Correspondencia: Soraya Araya Correo: sorayaraya@gmail.com

Conflicto de interés: Los autores declaran no poseer conflicto de interés.

Recibido: 21/03/2018. Aceptado: 28/04/2018.

DOI: https://doi.org/10.31698/ped.45012018003 


\section{INTRODUCCIÓN}

Varios estudios de investigación han recalcado la importante contribución de las infecciones bacterianas adquiridas en la comunidad en la morbilidad y mortalidad de los pacientes, si bien la mayoría de estos estudios se han llevado a cabo en población adulta, los pocos estudios realizados en niños, confirman el rol de las bacteriemias en la gravedad, sobre todo en menores de 5 años de edad ${ }^{(1)}$.

El espectro de patógenos causantes de bacteriemia varía según el estado inmunológico del paciente y la edad, en estudios conducidos antes de 1990, S.pneumoniae, Haemophilus influenzae Tipo B (HIB) y Neisseria meningiditis fueron causantes de bacteremia en un $75 \%, 20 \%$ y $5 \%$ respectivamente, con ocasional aislamiento de Salmonella y Staphylococcus aureus; luego de la introducción de la vacuna contra el Haemophilus influenzae las bacteremias por Haemophilus influenzae disminuyeron considerablemente hasta constituirse en un patógeno inusual $^{(2)}$.

Si bien en nuestro país las vacunas contra el Haemophilus influenzae tipo b (Hib) y el Streptococcus pneumoniae han sido introducidas al Calendario Nacional de Inmunización, la mortalidad infantil por causa infecciosa continúa siendo elevada y las bacteriemias por neumococo continúan siendo un problema.

En el año 2012 otro estudio identificó como agentes causantes de bacteriemia: S. aureus, Streptococcus pneumoniae (serotipos no incluidos en la vacuna 7 Valente), Neisseria meningitidis y Escherichia coli en población inmunocompetente y Pseudomonas aeruginosa y Candida en inmunocomprometidos ${ }^{(3)}$.

Referente a las particularidades de cada germen, es sabido que actualmente la bacteriemia adquirida en la comunidad causada por $S$. aureus es de frecuente observación en niños, con alta participación de cepas de $S$. aureus meticilino resistente; según las últimas publicaciones los pacientes con infecciones por $S$. aureus tienen alta morbilidad y un tercio de los niños afectados presenta focos de infección secundaria o metastásica (ej.: endocarditis, neumonía u osteomielitis), siendo la mortalidad elevada entre $15 \%$ y $40 \%$ en algunas series. En relación a este punto según estudios realizados en Nueva Zelanda y Canadá, la incidencia en niños de Bacteriemia por $S$. aureus adquiridas en la comunidad (BSAC), se estimó en 16,9 y 17,9 casos por cada 100000 personas, respectivamente. Siendo en un hospital pediátrico de Argentina 1,08 casos por cada 1000 ingresos hospitalarios ${ }^{(4)}$.

Faltan más estudios latinoamericanos que contemplen la bacteriemia en población pediátrica, analizando la etiología según grupos de edad y los factores de riesgos que inciden en la gravedad y la mortalidad. El conocimiento de la etiología por edad y de los factores de riesgo que inciden en la gravedad y mortalidad del paciente al momento del ingreso hospitalario, será de gran utilidad al momento de optar por Estrategias Preventivas de Salud Pública, las que tendrán un impacto positivo en el aumento de la sobrevida de estos niños.

\section{OBJETIVOS}

Identificar los agentes etiológicos y perfil de sensibilidad en Bacteriemias Adquiridas en la Comunidad, en pacientes pediátricos, así como también los factores de riesgos asociados a severidad y mortalidad.

\section{MATERIALES Y MÉTODOS}

Tipo de estudio: Estudio retrospectivo de todas las bacteriemias adquiridas en la comunidad, diagnosticadas en el hospital durante el período comprendido entre enero de 2007 y enero de 2015. Se tuvo en cuenta las variables demográficas, clínicas, evolutivas, laboratoriales y microbiológicas. Se identificó a los agentes infecciosos más frecuentes y el perfil de sensibilidad a antibióticos de los mismos, se estableció la asociación entre cada una de las variables y la severidad, así como también entre las variables y la mortalidad. Criterio de inclusión: pacientes con edad comprendida de 29 días de vida a 16 años de edad, con infecciones procedentes de la comunidad y aislamiento bacteriano en 
hemocultivo, con menos de 48 h de internación en el servicio, sin antecedente de internación previa.

Criterios de exclusión: menores de 29 días de vida, pacientes sin aislamiento de germen en hemocultivo, aislamientos considerados contaminantes: Staphylococcus Coagulasa-negativo en 1 solo hemocultivo, aislamiento de Corynebacterium spp, Bacillus spp y Streptococcus viridans(1/2), aislamiento de bacterias en hemocultivos en muestras tomadas posterior a 48 hs de internación.

\section{Definiciones:}

La Bacteriemia se definió como la presencia de hemocultivo positivo para bacterias gram positivas o gram negativas y signos compatibles con infección, obtenidas en las primeras 48 hs de internación.

Fiebre: temperatura axilar mayor o igual a $37.5^{\circ} \mathrm{Co}$ historia de fiebre dentro de las 24 horas de admisión Grupos de edad: lactante menor (29 dias-12 meses), lactante mayor (1-2 años) preescolar 2 años 1dia - 4 años, escolar (5-11años), adolescente (mayor o igual a 12 años).

Desnutrición severa cuando en niños menores de 2 años el peso para la edad se encontraba por debajo o igual a -3 DE, para mayores de 2 años utilizamos la tabla talla/edad.

La Anemia fue clasificada según la gravedad en leve, moderada y severa, utilizando los criterios de la OMS (Tabla 1).

Tabla1. Clasificación de la anemia según la edad

\begin{tabular}{lccc}
\hline Grupos de Edad & Anemia Leve & Anemia Moderada & Anemia Severa \\
\hline Niño de $\mathbf{6}$ meses a $\mathbf{5}$ años & $10,0-10,9$ & $7,0-9,9$ & $<7,0$ \\
\hline Niño de $\mathbf{5}$ a $\mathbf{1 1}$ años & $10,0-11,4$ & $7,0-9,9$ & $<7,0$ \\
\hline Niño de $\mathbf{1 2}$ a $\mathbf{1 4}$ años & $10,0-11,9$ & $7,0,9,9$ & $<7,0$ \\
Mujer a partir de $\mathbf{1 5}$ años & $10,0-11,9$ & $7,0-9,9$ & $<7,0$ \\
\hline Varón a partir de $\mathbf{1 5}$ años & $12,0-12,9$ & $9,0-11,9$ & $<9,0$ \\
\hline
\end{tabular}

Hipoglicemia severa fue definida por glicemia igual o menor a $2.2 \mathrm{mmol} / \mathrm{L}$ y no severa cuando se encontraba entre $2.3-3.2 \mathrm{mmol} / \mathrm{L}$.

La endocarditis fue definida conforme a los criterios de Duke.
Choque fue definido como: llenado capilar mayor a 2 segundos más hipotensión para la edad.

\section{Tratamiento}

El tratamiento fue considerado como discordante cuando el antibiótico administrado no era eficaz en base al antibiograma del patógeno hallado en los hemocultivos. Si el niño recibía más de un antibiótico en el tratamiento empírico y uno de ellos era útil para el patógeno hallado, se lo consideró como concordante.

Evolución: Se tomó en cuenta el desenlace final del paciente (Vivo o fallecido), desarrollo de complicaciones (Infecciones a distancia, choque, endocarditis, neumonía, falla multiorgánica).

El óbito fue atribuido a la infección cuando el niño tuvo hemocultivos positivos para $S$. aureus $u$ otra bacteria o cuando presentó signos persistentes de infección sistémica refractarios al tratamiento instituido (ej.: fiebre, leucocitosis, hipotensión, etc.) al producirse el deceso.

Microbiología: Las bacterias se identificaron mediante las pruebas microbiológicas manuales y automatizadas. La sensibilidad y resistencia antibiótica conforme al manual CLSI de uso actual.

Análisis estadístico. Los datos se expresaron como media aritmética. Un valor de p menor de 0,05 se consideró significativo. Todos los datos se almacenaron en un programa de computación con el programa informático Epi-info, versión 2000 (Centers for Disease Control, Atlanta, Georgia, EE.UU.). Los valores fueron expresados en base al total de datos disponibles. La significación estadística se calculó mediante la prueba de la Xi al cuadrado $\left(\chi^{2}\right)$ o la prueba exacta de Fischer para las variables nominales y la prueba t de Student o la de Wilcoxon para las variables numéricas. Se halló el valor p y se realizó elanálisis de momios, con $95 \%$ de IC.

\section{RESULTADOS}

Durante el periodo de estudio se registraron 187 casos de Bacteriemias adquiridas en la Comunidad 
(BAC), siendo la edad media de los pacientes $40 \pm 35$ meses y la relación M/F 1,4:1.

En cuanto a la edad fueron $<1$ año el 30,5\% (57/187), de 1 a 4 años 11 meses 49,2\% (92/187), de 5 a 10 años $15,5 \%$ (29/187) y mayores de 10 años 4,8 \% (9/187). Siendo los diagnósticos de ingreso: Neumonía (78\%), Infección de Piel y Partes Blandas (9\%), Infección a Múltiples focos (7\%), Meningitis (3,7\%) y Bacteriemia sin foco $2 \%$ (Tabla 3 ).

Las bacteriemias producidas por Gram Positivos fueron más frecuentes que las bacteremias por Gram Negativos: (171/187) y $(16 / 187) 91,4$ vs $8,6 \%(p<0,05)$, siendo los aislamientos mas frecuentes: Streptococcus pneumoniae, Staphylococcus aureus, Staphylococcus coagulasa negativo (2/2) y Klebsiella pneumoniae en un $46 \%, 34 \%, 7,5 \%$ y $2 \%$ respectivamente, la resistencia a la meticilina se observó en el $20 \%$ de las cepas de $S$. aureus. No se constató resistencia a la cefotaxima en las cepas de S. pneumoniae, $12,5 \%$ de las bacterias Gram negativas, fueron productoras de Betalactamasa de espectro extendido (Tabla 2).

Tabla 2 Gérmenes aislados en Niños con Bacteriemia Adquirida en la Comunidad.

\begin{tabular}{|lcc|}
\hline Germen & N & $\%$ \\
\hline Streptococcus pneumoniae & 87 & 46 \\
\hline Staphylococcus aureus & 64 & 34 \\
\hline *Staphylococcus coagulasa negativo (2/2) & 14 & 7,5 \\
\hline Klebsiellapneumoniae & 4 & 2 \\
\hline Haemophilusspp & 3 & 1,6 \\
\hline S pyogenes & 2 & 1 \\
\hline S. warnerii & 2 & 1 \\
\hline S. viridans & 2 & 1 \\
\hline Escherichia coli & 2 & 1 \\
\hline Acinetobacter baumanii & 2 & 1 \\
\hline Enterobacter cloacae & 2 & 1 \\
\hline Neisseria meningitidis & 1 & 0,5 \\
\hline Enterococcus faecium & 1 & 0,5 \\
\hline Sphingomonas paucimobilis & 1 & 0,5 \\
\hline Candida (Infección concomitante) & 3 & 1,6 \\
\hline Rtaphylococcus aureus (MR- Co) & & \\
\hline Streptococcus pneumoniae (Cefotaxima R) & 13 & 20 \\
\hline Gram Negativos Productores de BLEE & 0 & 0 \\
\hline *taphlococcus coagulasa negativo: Staphylococcus epidermidis y Staphylococcus hominis \\
\hline
\end{tabular}

En cuanto a la gravedad al ingreso 31 ptes $(16,5 \%)$ presentaron saturación de $\mathrm{O} 2<94 \%, 48$ ptes $(25,6 \%)$ presentaron choque, 19 ptes $(10 \%)$ presentaron Glasgow $<12,63$ ptes( 33,7\%) requirieron ingreso a UCI, la mortalidad de la serie fue $16,5 \%$, siendo significativamente mayor en aquellos pacientes con bacteriemias por Gram Positivos ( $p=0,001)$, al establecer una comparación entre menores de 5 años y mayores de 5 años, si bien, hubo mayor frecuencia de bacteriemia en niños menores de 5 años de edad $(\mathrm{p}=0,0000001)$, la mortalidad fue similar en ambos grupos $(\mathrm{p}=0,8)$ (Tabla 4$)$.

Las variables presentes al ingreso del paciente que se asociaron a mortalidad fueron: edad $<5$ años ( $p<0,05)$, Desnutrición $(p=0,02, R R=2,4$, IC95\%: 1,15), Infección a múltiples focos( $\mathrm{p}=0,002, \mathrm{RR}=3,2$, IC 95\%: 1,6-6,4), Choque $\mathrm{p}<0,001$, RR19, IC95\%:7,8-46), Glasgow $<12(p<0,001, R R=5,5$, IC 95\% :3,2-9,6), saturación de O2<94\% ( $\mathrm{p}=0,007$, RR 2, IC95\%: 1,1-4), $\mathrm{Hb} \leq 7$ ( $<<0,01, \mathrm{RR}: 11, \mathrm{IC} 95 \%$ : 6,8 a 19), presencia de leucocitos $>15000$, plaquetas $<100000(\mathrm{p}<0,001$, $\mathrm{RR}=4,4$, IC95\%: 2,4- 7,8), Bacteriemia por Gram Positivos ( $\mathrm{p}=0,001, \mathrm{RR}=4,9$, IC 95\%: 1,5-15) (Tabla 4)

Tabla 3. Características Demográficas, Clínicas y Laboratoriales de los Niños con Bacteriemia (BAC) asociadas a Severidad. $\mathrm{N}=187$

\begin{tabular}{|c|c|c|c|c|c|c|c|}
\hline Variable & $\mathrm{UCl}(63)$ & $\%$ & No UCI (124) & $\%$ & Valor $\mathrm{p}$ & OR & IC $95 \%$ \\
\hline Edad Media & $44+38$ & & $36+33$ & & & & \\
\hline Sexo Fem (80) & 22 & 34,9 & 58 & 46,8 & 0,15 & 0,61 & $0,3-1,1$ \\
\hline Sexo Masc (107) & 41 & 65,1 & 66 & 53,2 & & 1,64 & $0,8-3,0$ \\
\hline DDI (días promedio) & $16+13$ & & $12+10$ & - & - & - & - \\
\hline Prodromo (días promedio) & $5,5+5$ & & $10+6$ & - & - & - & - \\
\hline Duración de la Fiebre (dias) & 5) 4,5 & 7,1 & 2,7 & - & - & - & - \\
\hline Temperatura $\left({ }^{\circ} \mathrm{C}\right)$ & $38+0,8$ & & $36+2$ & - & - & - & \\
\hline Choque & 29 & 46,0 & 11 & 8,9 & 0,0000 & 8,76 & $3,9-19,3$ \\
\hline Glasgow $<12$ & 17 & 27 & 2 & 1,6 & 0,0000 & 22,54 & $5-101$ \\
\hline Sat $02<94$ & 44 & 69,8 & 8 & 6,5 & 0,0000 & 33,58 & $13,7-82$, \\
\hline Comorbilidad & 37 & 58,7 & 60 & 48,4 & 0,21 & 1,52 & $0,8-2,8$ \\
\hline Coinfeccion & 7 & 11,1 & 13 & 10,5 & 1 & 1,07 & $0,4-2,8$ \\
\hline Tto ATB previo & 12 & 19 & 8 & 6,5 & 0,01 & 3,41 & $1,3-8,8$ \\
\hline Tto discordante & 20 & 31 & 17 & 13,7 & 0,003 & 2,9 & $1,4-6,1$ \\
\hline \multicolumn{8}{|l|}{ Dx al ingreso } \\
\hline Neumonía (146) & 48 & 76,2 & 98 & 79,0 & 0,7 & 0,85 & $0,4-1,7$ \\
\hline IPPB (17) & 3 & 4,8 & 14 & 11,3 & 0,2 & 0,39 & $0,1-1,4$ \\
\hline Osteoarticular (4) & 1 & 1,6 & 3 & 2,4 & 1 & 0,65 & $0,06-6,3$ \\
\hline Meningitis (7) & 5 & 7,9 & 2 & 1,6 & 0,04 & 5,26 & $0,9-27,9$ \\
\hline Múltiples Focos (13) & 6 & 9,5 & 7 & 5,6 & 0,4 & 1,76 & $0,5-5,4$ \\
\hline Bacteriemia sin Foco (4) & 0 & 0,0 & 4 & 3,2 & 0,3 & 0,00 & - \\
\hline \multicolumn{8}{|l|}{ Laboratorio } \\
\hline GB & $8277+14695$ & & $14650+2379$ & - & - & - & - \\
\hline $\mathrm{GB}<5000$ & 9 & 14,3 & 9 & 7,3 & 0,18 & 2,13 & $0,8-5,6$ \\
\hline GB $>15000$ & 47 & 74,6 & 41 & 33,1 & 0,0000 & 5,95 & $3,0-11,7$ \\
\hline $\mathrm{Hb}$ & $9,9+1,5$ & & $9,9+0,8$ & - & - & - & - \\
\hline $\mathrm{Hb}<7$ & 28 & 44 & 27 & 17 & 0,001 & 2,9 & $1,5-5,5$ \\
\hline Trombocitopenia & 10 & 15,9 & 8 & 6,5 & 0,06 & 2,74 & $1,02-7,3$ \\
\hline Gram Positivos & 55 & 87,3 & 116 & 93,5 & 0,17 & 0,47 & $0,2-1,3$ \\
\hline Gram Negativos & 8 & 12,7 & 8 & 6,5 & & 2,11 & $0,7-5,9$ \\
\hline
\end{tabular}


Tabla 4. Características epidemiológicas, clínicas y laboratoriales de pacientes con bacteriemia asociadas con mortalidad.

\begin{tabular}{|c|c|c|c|c|c|c|c|}
\hline Variables & $\mathrm{N}=\mathbf{1 8 7}$ & $\%$ & $\begin{array}{c}\text { Muertos } \\
\mathrm{N}=31\end{array}$ & $\begin{array}{c}\text { Vivos } \\
N=156\end{array}$ & Valor $\mathrm{p}$ & RR & IC $95 \%$ \\
\hline Edad promedio (meses) & $40 \pm 35$ & 41,17 & $53 \pm 43,8$ & (1) & & & \\
\hline Sexo Femenino & 77 & 58,82 & 16 & 61 & 0,1 & 1,5 & $0,8-2,9$ \\
\hline Sexo Masculino & 110 & & 15 & 95 & & & \\
\hline Edad & & 30,5 & & & & & \\
\hline$<1$ año & 57 & 49,2 & 9 & 48 & & & \\
\hline 1 año a 4 años 11 meses* & 92 & 15,5 & 15 & 77 & & & \\
\hline 5- 10 años & 29 & 4,8 & 5 & 24 & & & \\
\hline$>10$ años & 9 & & 2 & 7 & & & \\
\hline DDIm & $14 \pm 10$ & & $11,4 \pm 21$ & $12,4 \pm 10$ & ns & & \\
\hline Pródromo & $8,8 \pm 6$ & & $7,4 \pm 6,6$ & $9,1 \pm 5,7$ & ns & & \\
\hline Hipotermia & 10 & 5,3 & 3 & 7 & 0,2 & 1,9 & $0,7-5$ \\
\hline $\mathrm{T}>38,5$ & 41 & 21,9 & 23 & 18 & 0,00000001 & 10,24 & $5-21$ \\
\hline Choque & 40 & 21,4 & 26 & 14 & 0,00000001 & 19,1 & $7,8-46$ \\
\hline Glasgow $\leq 12$ & 19 & 10,2 & 12 & 7 & 0,00000001 & 5,5 & $3,2-9,6$ \\
\hline Comorbilidad & 91 & 48,6 & 15 & 76 & 0,9 & 0,9 & $0,6-1,4$ \\
\hline Tto ATB previo & 20 & 10,7 & 6 & 14 & 0,08 & 2 & $0,9-4,3$ \\
\hline UTI & 63 & 33,7 & 31 & 32 & 0,00000001 & $\mathrm{nc}$ & $\mathrm{nc}$ \\
\hline ARM & 62 & 33,2 & 30 & 32 & 0,00000001 & 60 & $8,4-433$ \\
\hline Neumonía & 145 & 77,5 & 24 & 121 & 0,7 & 1,17 & $0,5-2,7$ \\
\hline IPPB & 17 & 9,1 & 1 & 16 & 0,2 & 0,35 & $0,1-2,4$ \\
\hline Infecc. Múltiples focos & 13 & 6,9 & 6 & 7 & 0,002 & 3,2 & $1,6-6,4$ \\
\hline Meningitis & 7 & 3,7 & 0 & 7 & 0,2 & $\mathrm{nc}$ & $\mathrm{nc}$ \\
\hline Bacteriemia sin foco & 4 & 2,1 & 0 & 4 & 0,3 & $\mathrm{nc}$ & $\mathrm{nc}$ \\
\hline $\mathrm{Hb} \leq 7$ & 28 & 15 & 27 & 1 & 0,00000001 & 38 & $14-101$ \\
\hline$G B \geq 15000$ & 43 & 23 & 14 & 29 & 0,0013 & 2,76 & $1,5-5$ \\
\hline $\mathrm{GB} \leq 5000$ & 85 & 45,5 & 6 & 79 & 0,0013 & 0,29 & $0,1-0,7$ \\
\hline Plaquetas $\leq 100000$ & 16 & 8,5 & 7 & 9 & 0,0000081 & 4,37 & $2,4-7,8$ \\
\hline $\mathrm{Ph} \leq 7$ & 18 & 9,6 & 17 & 1 & 0,0000001 & 11,4 & $6,8-19,1$ \\
\hline Sat $\mathrm{O} 2 \leq 94$ & 31 & 16,6 & 9 & 22 & 0,04 & 2,06 & $1,1-4$ \\
\hline Sat $\mathrm{O} 2 \leq 90$ & 26 & 13,9 & 9 & 17 & 0,007 & 2,53 & $1,3-4,9$ \\
\hline Aislamientos Gram Positivos & os 171 & 91,4 & 29 & 142 & 0,001 & 4,89 & $1,5-15$ \\
\hline Aislamiento Gram Negativo & $\operatorname{vos} 16$ & 8,6 & 3 & 13 & 0,8 & 1,2 & $0,4-3,4$ \\
\hline
\end{tabular}

\section{DISCUSIÓN}

En países Europeos, la incidencia de bacteriemias adquiridas en la comunidad (BAC) es de 3 casos por 1000 admisiones según publicaciones de Francia y de $7 \%$ de las admisiones hospitalarias en un Centro de Referencia del Reino Unido(5), mientras que según publicaciones de Latinoamérica representan el 10\% de las admisiones hospitalarias en Argentina y el Brasil $^{(6)}$. En cuanto a admisiones a UCIP en países desarrollados las bacteriemias adquiridas de la comunidad representan el $10 \%$ de las admisiones y en países no desarrollados el $30 \%$ de las mismas ${ }^{(7)}$. Watson et al revelaron las diferencias en la incidencia de (BAC) según la edad,siendo mayor en menores de 1 año (156/100000) comparada con 5-9 años $(22 / 100000)$ y $\left(20 / 100\right.$ 000) en adolescentes ${ }^{(8)}$, conforme a nuestra serie, las bacteriemias de origen comunitario fueron significativamente mayor en los pacientes menores de 5 años que en los mayores de 5 años $(\mathrm{p}<0,05)$. Siendo los diagnósticos de ingreso: Neumonía (78\%), Infección de Piel y Partes Blandas (9\%), Infección a Múltiples focos (7\%), Meningitis $(3,7 \%)$ y Bacteriemia sin foco $2 \%$ lo cual es de esperarse por la alta frecuencia de neumonía en países en vía de desarrollo y de infección de piel y partes blandas en países tropicales.

Resulta fundamental el conocimiento de la epidemiologia local al momento de instaurar el tratamiento antibiótico empírico inicial, es así que en relación a este aspecto existen artículos científicos de países desarrollados como el Reino Unido que reconocen como agentes causales de (BAC) en población pediátrica a Streptococcus pneumoniae, Neisseria meningitidis, Staphylococcus aureus, Escherichia coli, Klebsiella spp. (en un 19\%, 16\%, 15\%, $10 \%$ y 4,5\% respectivamente), Doit el al en Francia identificaron al S. pneumoniae como el agente causal principal, seguido por frecuencia de $E$ coli y $S$, aureus en un $27,5 \%, 21 \%$ y $12 \%$ respectivamente ${ }^{(9)}$, en otro estudio colaborativo multicéntrico que enfocó pacientes pediátricos de hospitales de Bangladesh, Bolivia, Ghana, India, Pakistán y Sud Africa identificaron al S. aureus y a los Bacilos Gram negativos como a las bacterias mayormente aisladas en hemocultivos ${ }^{(10)}$; en el año 2017 Neuman MI, Hall M et al en una serie de 7509 niños constataron que el $78 \%$ de los aislamientos en (BAC) correspondió al S. pneumoniae ${ }^{(11)}$.

Conforme al presente estudio del Instituto de Medicina Tropical las (BAC) producidas por Gram Positivos fueron más frecuentes que las bacteriemias por Gram Negativos 91,4 vs $8,6 \%$ ( $p<0,05)$, siendo los aislamientos más frecuentes: Streptococcus pneumoniae, Staphylococcus aureus, Staphylococcus coagulasa negativo (2/2) y Klebsiella pneumoniae en un $46 \%, 34 \%, 6 \%$ y $2 \%$ respectivamente, al hacer la comparación con los artículos mencionados en el párrafo anterior resulta indudable la participación del S pneumoniae y del S.aureus en la génesis de la bacteriemia de origen comunitario, si bien en nuestra casuística no se hallaron cepas de Spn resistentes a cefotaxima y se hallaron $20 \%$ de cepas de $S$. aureus $M R$, estos hallazgos resultan útiles al momento de establecer el tratamiento antibiótico empírico inicial.

En cuanto a la etiología de la bacteriemia adquirida en la comunidad según la edad, conforme a una 
publicación del año 2018 de Korea, en menores de 3 meses de edad los agentes mas frecuentes fueron Escherichia coli (41\%), Streptococcus agalactiae $(27,7 \%$ )y S. aureus (27\%), de 3 meses a 59 meses Streptococcus pneumoniae ( $54 \%$ ), S aureus (20\%), Salmonella (14,4\% ) y en mayores de 5 años $S$ aureus (63\%), Salmonella $(12,4 \%)$, S. pneumoniae $(11,5 \%)$, con similares hallazgos en Francia, Australia y Reino Unido, en todos ellos se evidenció la participación de bacterias Gram negativas en menores de 3 meses, de $S$. pneumoniae en niños de 3 meses a 5 años y del $S$. aureus en mayores de 5 años ${ }^{(12)}$.

Según un metanálisis llevado a cabo en Latinoamérica,la incidencia de bacteriemia por $S$. pneumoniae en niños de 0-59 meses de edad fue 3.9/100 000 (95\% CI: 2.0-5.9) tomando en cuenta para la estimación de dicha incidencia la información de 3 países (Argentina, Chile y Costa Rica). Siendo los serotipos $14 \quad(29.3 \%$ de las muestras) (95\% CI: 24.5-34.3) seguido por el serotipo 6B, 10.6\% (95\% CI: $7.2-14.5)$ y serotipo $19 \mathrm{~F}$ in $7.3 \%$ (95\% CI: 5.5-9.0. La mortalidad en bacteremias por neumococo fue $8.3 \%$. Llegando a la conclusión que las meningitis y bacteriemia por S. pneumoniae son la causa mas importante de mortalidad en niños menores de 5 años de edad ( Ciapponi et al) ${ }^{(13)}$. Este hallazgo de predominancia de infecciones severas por neumococo se observó en todos los grupos de edad de nuestra serie con mayor participación del $S$. aureus en los niños mayores de 5 años de edad.

Si bien el presente trabajo no se enfocó en los serotipos de neumococo circulantes en bacteriemia ni en la biología molecular del S. aureus en (BAC), resulta de valor los hallazgos epidemiológicos considerando que en Paraguay en el año 2012 se ha implementado en el Calendario Nacional de Inmunización, la vacunación antineumocóccica en un esquema de $(2+1)$ con la vacuna conjugada antineumocóccica 10 Valente ${ }^{(14)}$.

$\mathrm{Al}$ valorar las variables clínicas y laboratoriales que inciden en la mortalidad en niños con (BAC), en relación a la edad, si bien la presencia de (BAC) fue significativamente mayor en los menores de 5 años de edad, la mortalidad fue semejante en ambos grupos $(\mathrm{p}=0,8)$, al establecer la relación entre las demás variables, la presencia de desnutrición, la infección a múltiples focos, la presencia de choque al ingreso, Glasgow $<12$, Saturación de Oxígeno menor a $94 \%$, anemia severa y bacteriemia por Gram positivos se asociaron significativamente a mortalidad en la presente serie; siendo la mortalidad de la misma $16,5 \%$ similar a la mortalidad por (BAC) registrada en Estados Unidos y Europa 15 a 22\% y menor a la mortalidad registrada en otros Países de Latinoamérica 28 a $40 \%{ }^{(15,16,5)}$

El presente trabajo posee sus limitaciones por ser un trabajo retrospectivo, es por ello que actualmente estamos llevando a cabo una serie prospectiva con el seguimiento en el tiempo de la epidemiología de las (BAC), tomando en cuenta los serotipos de las cepas de neumococo aisladas, así como también el perfil de sensibilidad antimicrobiana de los demás gérmenes Gram positivos y Gram negativos, lo cual nos orientará en las futuras modificaciones de pautas de tratamiento empírico e introducción local de vacunas. Si bien hasta la fecha no se ha licenciado una vacuna para uso humano para la prevención de Infecciones Severas por S.aureus, publicaciones recientes mencionan a la vacuna de Pfizer SA4Ag, como posible candidata por contar con estudios de fase más avanzada, la misma está compuesta por cuatro antígenos: la molécula de adhesión ClfA, el transportador de manganeso MntC y polisacáridos capsulares anti-fagocíticos 5 y $8^{(17)}$. Esta combinación está diseñada para provocar respuestas inmunes amplias humorales y celulares contra múltiples mecanismos de virulencia involucrados en el establecimiento y mantenimiento de la infección, pero hasta la fecha los estudios piloto solo han enfocado su uso para población adulta ${ }^{(17)}$

Finalmente cabe señalar, que en niños con (BAC), medidas tan simples como el Control de la Saturación de Oxígeno al ingreso, la administración oportuna del Oxigeno suplementario, el uso adecuado de hemoderivados en la anemia severa y de los fluídos en caso de que el paciente estuviera en choque, junto con la instauración de un tratamiento antibiótico oportuno que considere la participación del S. pneumoniae y del S.aureus (MR-Co), permitirán mejorar la sobrevida de los pacientes. 


\section{CONCLUSIÓN}

En niños con infecciones provenientes de la comunidad son más frecuentes las bacteriemias por Gram positivos,los agentes causales más frecuentes fueron el Streptococcus pneumoniae y el Staphylococcus aureus, $20 \%$ de los aislamientos de $S$. aureus fueron meticilino resistentes. Las bacteriemias por Gram
Positivos se asociaron a mayor severidad.

Se identificaron factores de riesgos asociados a mortalidad: edad $<5$ años, desnutrición, infección a múltiples focos, choque, glasgow $<12$, Sat O $2<94 \%$, $\mathrm{Hb}<7$, glóbulos blancos $>15000$, plaquetas $<100000$, Bacteriemia por Gram Positivos.

\section{REFERENCIAS}

1. Vaccine preventable deaths and the Global Immunization Vision and Strategy, 2006-2015. MMWR Morb Mortal Wkly Rep. 2006;55(18):511-515

2. O'Brien KL, Wolfson LJ, Watt JP, et al. Burden of disease caused by Streptococcus pneumoniae in children younger than 5 years: global estimates. Lancet. 2009;374(9693): 893-902.

3. Dat, HieuNgocVu, HungNguyenThe, HoaThiNguyen, Long BaoHoang, DungVuTienViet, Chi Linh Bui1, Kinh Van Nguyen, TrungVuNguyen, DaoTuyetTrinh, Alessandro Torre, H. Rogier van Doorn, BehzadNadjm and Heiman FL. Wertheim. Bacterial bloodstream infections in a tertiary infectious diseases hospital in Northern Vietnam: aetiology, drug resistance, and treatment outcome BMC Infectious Diseases. 2017;17:493

4. Pérez G, Martirena S, Reijtmanb V, Romerob R, Mastroiannib A, Casimirb L,Bologna R. Bacteriemia por Staphylococcusaureus adquirido en la comunidad en niños: estudio de cohorte 2010-2014.Arch Argent Pediatr 2016;114(6):508-513

5. Doit C, Kurkdjian M, Message M, Bidet P, Bonacorsi, Agnes C, Varon ES,Bingen E. Epidemiology of pediatric community acquired bloodstream infections a children hospital in Paris France, 2001 to 2008. Diagnostic Microbiology and Infectious Disease 2010; 66(3):332-335

6. De la Rosa G, León AL, Jaimes F. Epidemiología y pronóstico de pacientes con infección del torrente sanguine en 10 Hospitales de Colombia. Rev Chilena Infectol 2016;33(2): 141-149

7. Irwin AD, Drew RJ, Marshall P, Nguyen K, Hoyle E, Macfarlane KA, Wong HF,Mekonnen E, Hicks M, Steele T, Gerrard C, Hardiman F, McNamara PS, Diggle PJ, Carrol ED. Etiology of Childhood Bacteremia and Timely Antibiotics Administration in the Emergency Department. Pediatrics 2015; 135(4)
8. Pai S, Enoch DA, Aliju SH. Bacteremia in children: epidemiology, clinical diagnosis and antibiotic treatment Expert Rev Anti Infect Ther. 2015;13(9):1073-88

9. Jaramillo-Bustamante JC, Marın-Agudelo A, Fernandez-Laverde $\mathrm{M}$, et al. Epidemiology of sepsis in pediatric intensive care units: first Colombian multicenter study. PediatrCrit Care Med 2012;13:501-8

10. Rhie K, Choi EH, Cho EY, Lee J, Kang JH,Kim DS, et al. Etiology of Invasive Bacterial Infections in Immunocompetent Children in Korea (2006-2010): a Retrospective Multicenter Study. J Korean Med Sci. 2018;33(6):e45

11. Neuman MI, Hall M, Lipsett SC, Hersh AL, Williams DJ, Gerber JS, et al. Utility of Blood Culture Among Children Hospitalized With Community-Acquired Pneumonia. Pediatrics.2017; 140(3):e20171013.

12. National Institute of infectious diseases. Infectious agents: surveillance report. Internet. Japón: IASR; 2017. Citado el 30 de abril de 2017. Recuperado a partir de:http://www.niid.go.jp/niid/en/iasr-e.html.

13. Ciapponi A,Elorriaga N, Rojas JI, Romano M,GarcíaMartí S, Bardach A,Ruvinsky S. Epidemiology of Pediatric Pneumococcal Meningitis and Bacteremia in Latin America and the Caribbean A Systematic Review and Meta-analysis. The Pediatric Infectious Disease Journal 2014;33(9):971-978

14. Ministerio de Salud Pública y Bienestar Social. Lineamientos Técnicos y Operativos para la Vacunación contra Neumococo Vacuna de neumococo 10-valente conjugada. PCV10 Programa Ampliado de Inmunizaciones; 2012.

15. Delport JA, Strikwerda A, Armstrong A, Schaus D, John M. MALDI-ToF short incubation identification from blood cultures isassociated with reduced length of 
hospitalization and a decrease in bacteremia associated mortality. Eur J ClinMicrobiol Infect Dis 2017; 36(7):11811186

16. Laupland KB. Incidence of bloodstream infection: a review of population-based studies. Clinical
Microbiology and Infection. 2013; 19(6):492-500

17. GiersingBirgitte, Dastgheyb Sana, ModjaeedKayvon, MorthyVasee. Status of vaccine research and developmentof vaccines for Staphylococcus aureus. Vacine 2016;34(26) 2962-2966. 